\title{
Organic Electronic Device Modeling at the Nanoscale
}

\author{
Conor Madigan \\ MIT, Lab of Organic Optics and \\ Electronics \\ 77 Mass. Ave., Rm. 13-3138 \\ Cambridge, MA 02139 \\ 001-617 253-3181 \\ cmadigan@mit.edu
}

\author{
Vladimir Bulović \\ MIT, Lab of Organic Optics and \\ Electronics \\ 77 Mass. Ave., Rm. 13-3138 \\ Cambridge, MA 02139 \\ 001-617 253-7012
}

\begin{abstract}
Electronic devices with nanoscale features $(\sim 100 \mathrm{~nm}$ or smaller $)$ are becoming increasingly important in electronics technology. While nanoscale electronic devices comprise a variety of different material sets and structures, many of the nanoscale devices developed in the last decade employ organic materials. In this talk, we discuss the modeling of organic electronic thin film devices. In our approach, analysis of such devices begins on the molecular scale, and device level behavior is then derived from the combination of individual molecular properties and physical models of intermolecular interactions. We present a general purpose Monte Carlo simulator based on molecular-scale physical models and employ this simulator to analyze device behavior.
\end{abstract}

\section{Categories and Subject Descriptors} I.6.3 [Simulation and Modeling]: Applications.

\section{General Terms}

Theory.

\section{Keywords}

Nanoscale, organic, device modeling, molecular.

\section{INTRODUCTION}

Analysis of the optical and electronic behavior of organic electronic devices requires development of molecular (nanometer) scale physical models. In this work we present an approach to a complete organic electronic device simulator, and demonstrate application of the simulator to modeling the physical behavior of organic electronic devices.

Permission to make digital or hard copies of all or part of this work for personal or classroom use is granted without fee provided that copies are not made or distributed for profit or commercial advantage and that copies bear this notice and the full citation on the first page. To copy otherwise, to republish, to post on servers or to redistribute to lists, requires prior specific permission and/or a fee.

ICCAD'06, November 5-9, 2006, San Jose, CA

Copyright 2006 ACM 1-59593-389-1/06/0011...\$5.00

\section{Organic Electronic Devices}

Over the last two decades, a wide variety of organic electronic devices have been developed including light emitting devices[1,2], solar cells[3], photodetectors[3], and thin film transistors[4]. In all of these devices, the key electronic excitations are charge carriers (i.e. "electrons" and "holes") and excitons (i.e. bound electron-hole pairs), typically confined to individual molecules. In addition, the device active layers are almost universally between 1 and $100 \mathrm{~nm}$ in thickness. The nanometer scale of the device layers gives rise to a set of nanoscale phenomena that are easily identifiable in molecular device structures. For example, frequently the electronic properties of the organic thin films are controlled by molecularscale disorder (see e.g. [5,6]). This is most apparent in amorphous thin films in which the molecules are randomly positioned and oriented, but also applies to polycrystalline films. Furthermore, it is found that nanoscale interactions at heterojunctions and contacts further influence device behavior. Thus the analysis of molecular and polymeric organic electronic devices represents a fundamentally nanoscale challenge.

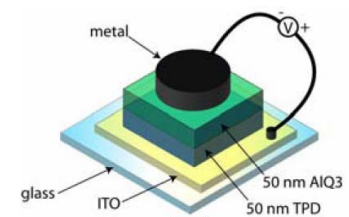

(a)

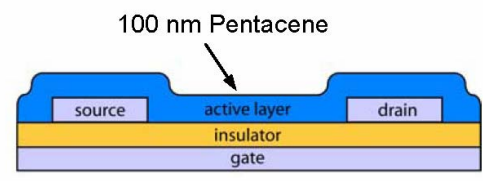

(b)
Figure 1. Examples of organic electronic devices: (a) organic light emitting device; (b) organic thin film transistor.

\section{Nanoscale Simulation: From Molecules to Devices}

We have developed a Monte Carlo simulator from a molecularscale perspective. The principle components of the simulator are: (1) the molecular types, positions, and orientations; (2) the intermolecular interactions that govern the relevant electronic processes (e.g. charge carrier transport, exciton diffusion, exciton dissociation, etc.); and (3) any intrinsic properties of each molecular type needed to compute the relevant interactions. This Organic and Nanostructured Electronics Simulator (ONESim) is a modular and extensible numerical tool, supporting a wide range of existing physical models and easily modified to support additional ones. 

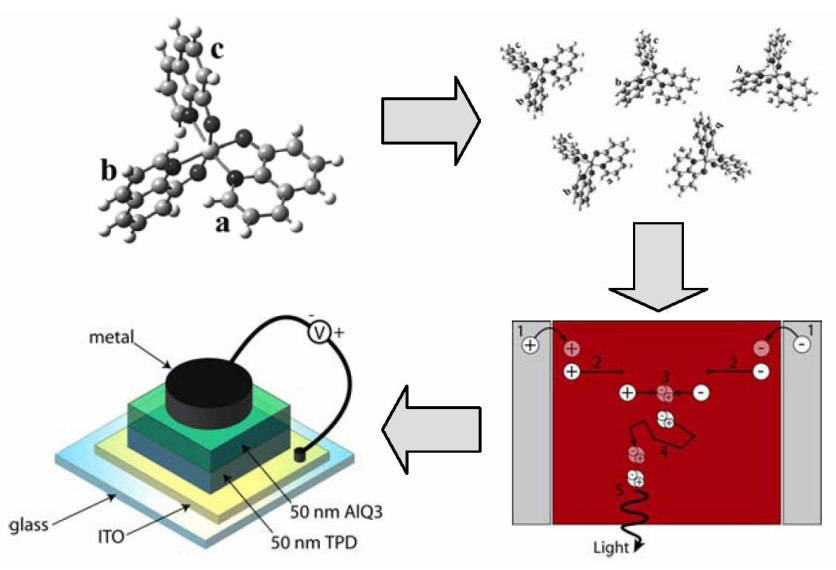

Figure 2. Overview of the ONESim simulation approach, from individual molecules to intermolecular interactions to bulk film properties to device behavior.

We have employed ONESim to analyze charge carrier transport and exciton diffusion in amorphous organic thin films, yielding calculations of the charge carrier mobilities and exciton diffusion lengths. These simulations reflect the complexity of even such basic parameters in organic electronic materials, where charge carrier mobilities are strongly field and carrier concentration dependent, and exciton diffusion is highly dispersive. We have also applied ONESim to the calculation of currents through metal/organic/metal stacks to obtain macroscopic I-V behavior through organic films of nanoscale thickness.

\section{Conclusion}

The rapid growth of organic electronics technology has to some extent outpaced the development of accurate physical models of device behavior. The approach described here, in which we combine Monte Carlo simulation techniques with molecular-scale physical models, shows great promise as a tool for organic electronic device analysis.

\section{ACKNOWLEDGMENTS}

This work was supported by an NSF CAREER Grant, an NDSEG Grant, and the MARCO MSD Focused Research Center.

\section{REFERENCES}

[1] C.W. Tang and S.A. VanSlyke, Appl. Phys. Lett., 51, 913 (1987).

[2] M.A. Baldo et al., Nature, 395, 151 (1998).

[3] P. Peumans, A. Yakimov, and S.R. Forrest, J. Appl. Phys., 93, 3693 (2003).

[4] C.D. Dimitrokopolous and P.R.L. Malenfant, Adv. Mat., 14, 99 (2002).

[5] H. Bassler, Phys. Stat. Sol. (b), 175, 15 (1993).

[6] C.F. Madigan and V. Bulović, Phys. Rev. Lett., 96, 046404 (2006). 